\title{
BMjNeurology Open Parkinsonism in the psychiatric setting: an update on clinical differentiation and management
}

\author{
Alice Powell (D , , ${ }^{1,2}$ Lara Gallur, ${ }^{3,4}$ Leslie Koopowitz, ${ }^{4,5}$ Michael William Hayes ${ }^{1}$
}

To cite: Powell A, Gallur

L, Koopowitz L, et al.

Parkinsonism in the psychiatric setting: an update on clinical differentiation and management. BMJ Neurology Open 2020;2:e000034. doi:10.1136/ bmjno-2019-000034

Received 21 December 2019 Revised 07 January 2020 Accepted 07 January 2020
Check for updates

(C) Author(s) (or their employer(s)) 2020. Re-use permitted under CC BY-NC. No commercial re-use. See rights and permissions. Published by BMJ.

${ }^{1}$ Department of Neurology, Concord Repatriation General Hospital, Sydney, New South Wales, Australia

${ }^{2}$ Brain and Mind Centre, The University of Sydney, Sydney, New South Wales, Australia ${ }^{3}$ Department of Psychiatry, Northern Adelaide Local Health Network, Adelaide, South Australia, Australia

${ }^{4}$ School of Medicine, Discipline of Psychiatry, The University of Adelaide, Adelaide, South Australia, Australia

${ }^{5}$ Brain Injury Rehabilitation Unit, Hampstead Rehabilitation Centre, Adelaide, South Australia, Australia

Correspondence to

Dr Alice Powell;

alice.powell@sydney.edu.au

\section{ABSTRACT}

Parkinsonism is seen frequently in patients with psychiatric conditions. Drug-induced parkinsonism (DIP) is the second most common cause of parkinsonism in the general population after Parkinson's disease (PD) but a range of rarer aetiologies, some of them reversible, should also be considered in patients of all ages. DIP is more common in older patients, as are neurodegenerative diseases that may produce parkinsonism and it is relatively more likely that drug exposure could be unmasking an underlying process in this population. There is an extensive literature on differentiating DIP from PD but clinical features can be indistinguishable and many proposed investigations are not readily available. Aside from cessation of the responsible medication, there is no clear consensus on treatment strategies or duration of treatment. Practically, a delicate balance must be achieved between ameliorating parkinsonism and avoiding recurrent psychosis. Long-term prognosis in the setting of DIP remains unclear. We review the features that may differentiate DIP from other causes of parkinsonism in patients with psychiatric illness, provide an update on relevant investigations and discuss management strategies. The use of atypical antipsychotics for a broad range of indications highlights the ongoing relevance of DIP.

\section{INTRODUCTION}

Parkinsonism, defined as bradykinesia in combination with either rest tremor, rigidity or both, ${ }^{1}$ classically arises in the context of Parkinson's disease (PD). The major pathology is represented by Lewy bodies containing alpha-synuclein and associated dopamine cell loss in the substantia nigra leading to dysfunction of basal ganglia circuitry responsible for motor control. However, parkinsonism due to a wide range of possible causes also occurs in up to onethird of patients taking antipsychotic medications. $^{2}$ This syndrome may significantly impair function and quality of life.

\section{Pathophysiology of parkinsonism}

In PD, it is proposed that there is an imbalance of activation of excitatory and inhibitory pathways involving the basal ganglia (a collection of subcortical grey matter nuclei including the substantia nigra, globus pallidus, subthalamic nucleus and the striatum comprising the putamen and caudate nucleus), thalamus and motor cortex governing motor output. ${ }^{34}$ Dopamine usually has an excitatory influence on the direct pathway via D1 receptors to facilitate cortically initiated movements and an inhibitory influence on the indirect pathway via $\mathrm{D} 2$ receptors, which usually impedes voluntary movement through thalamic inhibition. ${ }^{3}$ This pathway is summarised in figure 1.

In the case of PD, gradual degeneration of dopaminergic substantia nigra pars compacta neurons and their projections to the striatum leads to motor symptoms. ${ }^{3}$ A range of dopaminergic medications that either increase dopamine levels through a variety of mechanisms or stimulate dopamine receptors are used to address these symptoms. Conversely, any medication that either blocks dopamine D2 receptors or reduces dopamine release may result in parkinsonism. ${ }^{5}$ Other aetiologies of parkinsonism typically produce motor symptoms through damage to the basal ganglia, for example, through vascular injury in the case of vascular parkinsonism or normal pressure hydrocephalus.

A degree of parkinsonism is frequent in the elderly and may be due to an agerelated decline in the number of nigrostriatal neurons. ${ }^{67}$ However, neurodegenerative pathologies also accumulate with age. A recent study of over 1400 patients followed up for an average 8.5 years until death demonstrated that the majority $(94.1 \%)$ had at least one pathology on autopsy, the average individual had three pathologies and 25\% had PD pathology. ${ }^{8}$ The other pathologies detected included Alzheimer's pathology, cerebral amyloid angiopathy, cerebrovascular disease, hippocampal sclerosis and TAR DNA-binding protein $43 .{ }^{8}$ A majority $(53.9 \%)$ of this cohort had developed parkinsonism by their last visit. ${ }^{8}$ Interestingly, this study also 


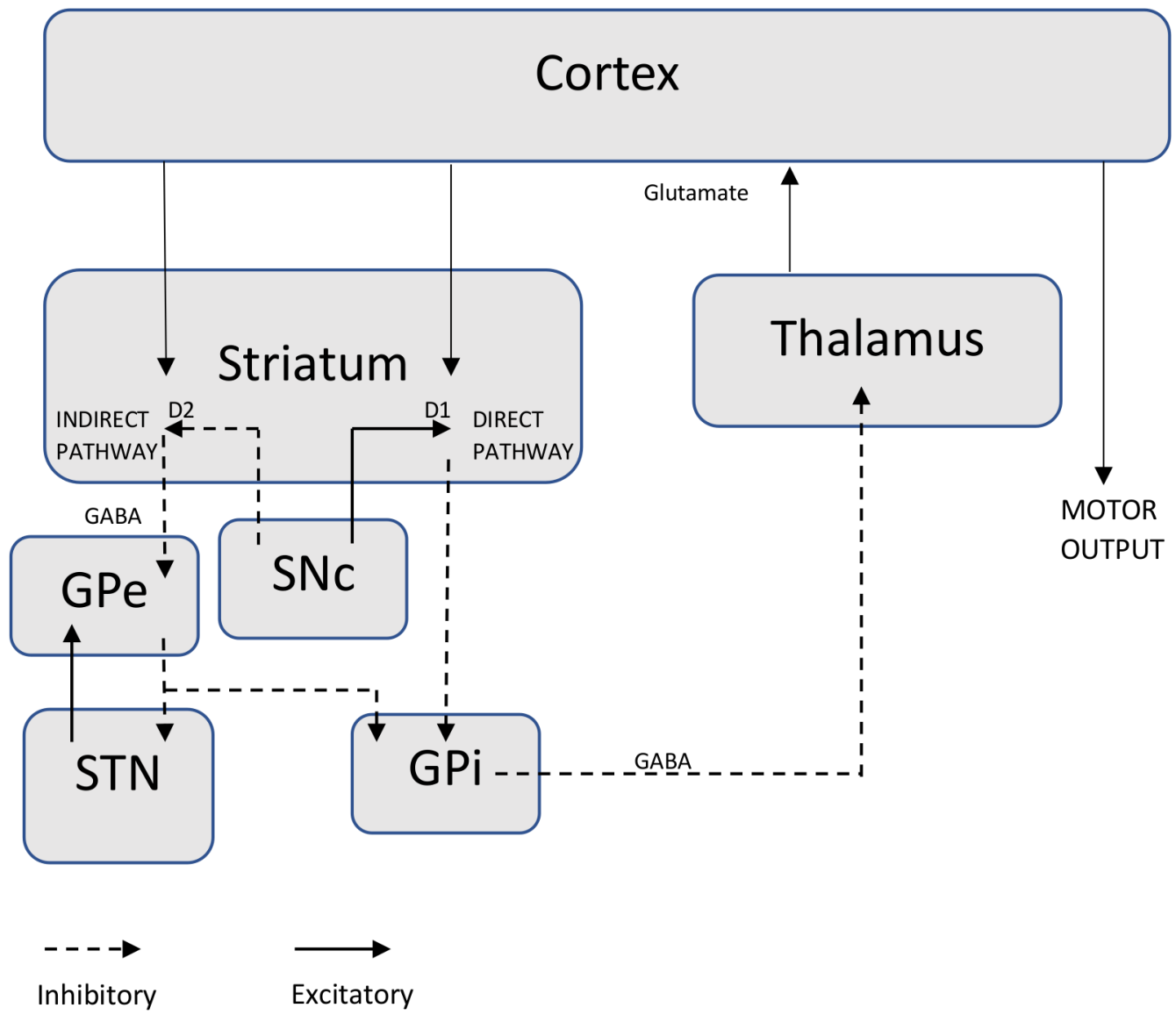

(GABA, D2)

(glutamate, D1)

Figure 1 Schematic diagram of excitatory and inhibitory basal ganglia pathways implicated in parkinsonism. Dopaminergic projections from the SNc have an excitatory influence on direct pathway striatopallidal fibres via D1 receptors leading to disinhibition of thalamic nuclei and enhanced thalamocortical drive facilitating cortically initiated movements. Dopamine also has an inhibitory effect on indirect pathway striatopallidal fibres via D2 receptors, which impedes voluntary movement through thalamic inhibition. The direct pathway is also activated by glutamatergic projections from the sensorimotor cortex and the indirect pathway via gamma-aminobutyric acid (GABA) from the putamen. GPe, globus pallidus externa; GPi, globus pallidus interna; SNc, substantia nigra pars compacta; STN, subthalamic nucleus.

highlighted that a proportion of patients with both PD and other pathologies did not demonstrate any clinical parkinsonism. $^{8}$

\section{Beyond dopamine: the role of other neurotransmitters in parkinsonism and psychosis}

Other neurotransmitter systems are also implicated in PD motor symptoms and drug-induced parkinsonism (DIP) and may help explain why DIP may occur with medications that do not have direct effects on dopamine levels or receptors. For example, due to the interrelationship between dopaminergic and cholinergic pathways, parkinsonism has been conceptualised as resulting from relative dopamine deficiency and relative acetylcholine excess. ${ }^{9}$ This hypothesis is supported by observations that parkinsonism is aggravated by centrally acting cholinergic medications and improved by anticholinergic medications. ${ }^{10}$ Gait impairment in PD is pathophysiologically complex and freezing of gait, specifically, may be associated with dysfunction in the noradrenergic and cholinergic systems. ${ }^{4}$
Similarly, contemporary understanding of neurotransmitter systems involved in psychosis has evolved beyond dopamine dysregulation with multiple other neurotransmitters associated with the syndrome. There is evidence that a relative hypocholinergic state favours the emergence of psychotic symptoms in PD. ${ }^{11}$ PD psychosis has also been conceptualised to reflect an imbalance between dopamine and serotonin neurotransmission. ${ }^{12}$ Atypical antipsychotics, such as quetiapine and clozapine, demonstrate significant serotonin receptor antagonism. ${ }^{13}$ This serotonin receptor activity differentiates typical from atypical antipsychotics and was touted as resulting in lower extrapyramidal side effects (EPSE) such as parkinsonism both due to the downstream effect on dopamine release and the need for a lower D2 receptor occupancy for antipsychotic efficacy. With the ongoing use of atypical antipsychotics, it has been demonstrated that there is still a risk of causing EPSE, and the 'atypical' nature may be lost with dose increases. 


\section{Drug-induced parkinsonism}

DIP is defined as the presence of parkinsonism without a history of parkinsonism before the use of the offending drug and onset of parkinsonian symptoms during use of the drug. ${ }^{5}$ DIP is relevant to patients to a broad range of patients since antipsychotic agents are prescribed for a range of psychiatric indications including adjunctive therapy for depression, anxiety disorders, personality disorders and behavioural and psychological symptoms of dementia. ${ }^{14}$ This perhaps reflects attitudes that antipsychotic medications are less addictive than, for example, benzodiazepines and may even be perceived as safer. It is possible that patients prescribed these medications for other indications are not properly appraised of the potential risks. ${ }^{14}$

While antipsychotic medication is a common cause of DIP, other medications have been causally associated with parkinsonism including antiemetic agents, ${ }^{5}$ antidepressants (selective serotonin reuptake inhibitors), ${ }^{15}$ lithium $^{16}$ and calcium channel blocking agents. ${ }^{5}$

Although DIP is less frequent with atypical antipsychotics than typical agents such as haloperidol, ${ }^{17}$ it is still commonly seen. A recent Cochrane review concluded that DIP has been associated with all second-generation antipsychotics. $^{18}$

There is significant individual variability in the propensity to develop parkinsonism in patients treated with causally associated medications which can be only partially explained by reported polymorphisms in genes involved in dopamine transmission in patients who develop DIP, ${ }^{19}$ or unmasking of subclinical PD or an atypical parkinsonian syndrome. ${ }^{20}$ Patients with dementia with Lewy bodies (DLB), a closely related synucleinopathy characterised by early cognitive symptoms, are exquisitely sensitive to the effects of dopamine receptor blocking agents. ${ }^{21}$

Treatment with a dopamine receptor blocker or dopamine depleting agent in a dose or time course consistent with DIP is an exclusion criterion for $\mathrm{PD}^{1}$ and tardive parkinsonism can persist for months or years after cessation of a dopamine receptor blocking agent. ${ }^{22}$ However, patients who continue to demonstrate parkinsonian features long after causative medications are ceased are thought more likely to have subclinical PD unmasked by neuroleptic exposure. ${ }^{23}$

\section{CLINICAL FEATURES AND DIFFERENTIAL DIAGNOSIS}

Differentiating DIP from PD or another underlying neurodegenerative disease is difficult. Both conditions are more frequent with advancing age, ${ }^{52}$ with PD affecting approximately $1 \%$ of the population over $60 .^{24}$ DIP is more common in women ${ }^{517}$ while PD has a slight male predominance. ${ }^{24}$

Clinical features found to be useful in identifying DIP include a clear temporal association with commencement of potentially responsible medication, bilateral and symmetric features, rigidity, relative absence of rest tremor and the coexistence of tardive dyskinesia. ${ }^{525}$ Freezing of gait is infrequent in DIP compared with other causes of parkinsonism. ${ }^{26}$ Non-motor symptoms, such as late-onset rapid eye movement sleep behaviour disorder, reflect the involvement of other neurotransmitter systems and are less likely to be a feature of DIP. ${ }^{20}$ Hyposmia, now part of the supportive diagnostic criteria for PD due to degeneration of the olfactory system, ${ }^{1}$ can be non-specific, particularly in older patients but is less frequent in DIP. ${ }^{20}$ Response to levodopa is diagnostically useful with a lack of response or less pronounced response typically seen in DIP. ${ }^{25}$ Assessing motor response objectively using wearable devices is becoming increasingly useful. ${ }^{27}$

When parkinsonism develops acutely or progresses rapidly and is associated with other neurological symptoms and signs, other rarer aetiologies should be considered. These include infective and autoimmune encephalitides, structural lesions, stroke and metabolic and genetic disorders. ${ }^{28}$ Rare secondary causes and investigations can be reviewed in table 1 .

\section{INVESTIGATIONS}

A number of ancillary investigations have been proposed to differentiate DIP from neurodegenerative pathology. Single-photon emission CT using (123) I-ioflupane as a dopamine transporter ligand (DaT-Scan) is a reliable technique for distinguishing between DIP and $\mathrm{PD}^{29}$ but is not readily available in many countries including Australia. DaT uptake in the striatum is significantly decreased in patients with PD even in early stages of the disease, and drugs causing parkinsonism including antipsychotics have negligible affinity for DaT, so scans may demonstrate normal uptake even with significant DIP. ${ }^{5}$

Another potentially useful auxiliary investigation is measurement of cardiac sympathetic denervation, an early feature of PD, using metaiodobenzylguanidine scintigraphy, ${ }^{30}$ but it is not funded as a test for PD in all countries.

MRI and transcranial sonography of the substantia nigra $^{31} 32$ can demonstrate dopamine depletion with absence of the 'swallow-tail' appearance of the substantia nigra and increased iron concentrations, respectively, but the specificity of these tests is uncertain and availability limited. ${ }^{31} 32$

Finally, a recent pilot study of the utility of cerebrospinal fluid neurofilament light (a biomarker of neuroaxonal damage) in the neuropsychiatric setting found that levels were significantly higher in patients with a neurological or neurodegenerative disorder compared with psychiatric disorder. ${ }^{33}$ In summary, these tests are promising but still not readily available and clinicians still need to rely largely on clinical acumen.

\section{MANAGEMENT STRATEGIES}

The treatment of parkinsonism in the psychiatric setting should be individualised, considering varied vulnerabilities and priorities for medication use. Where DIP 
Table 1 Investigations to exclude secondary causes of parkinsonism in atypical cases ${ }^{28} 40$

\begin{tabular}{|c|c|c|}
\hline Category & Test & Rationale/differential \\
\hline $\begin{array}{l}\text { Blood } \\
\text { tests }\end{array}$ & $\begin{array}{l}\text { Serum electrolytes, caeruloplasmin, thyroid } \\
\text { function tests, parathyroid hormone, blood film, } \\
\text { HIV and syphilis serology, autoantibodies (NMDAR, } \\
\text { LGI1, CRMP5, Ri, DPPX, Ma2) }\end{array}$ & $\begin{array}{l}\text { Wilson disease, hyper and hypothyroidism, } \\
\text { hypoparathyroidism and pseudohypoparathyroidism, } \\
\text { neuroacanthocytosis, HIV and neurosyphilis, autoimmune } \\
\text { encephalitis }\end{array}$ \\
\hline Urine & 24 hour urine copper & Wilson disease \\
\hline CSF & Viral PCR, syphilis, autoantibodies, 14-3-3 & $\begin{array}{l}\text { Encephalitis, neurosyphilis, autoimmune encephalitis, prion } \\
\text { disease }\end{array}$ \\
\hline \multirow[t]{2}{*}{ Imaging } & MRI brain & $\begin{array}{l}\text { Structural brain lesions (normal pressure hydrocephalus, } \\
\text { chronic subdural haematoma, tumours), stroke, evidence of } \\
\text { previous head trauma, encephalitis, manganese intoxication, } \\
\text { specific atrophy patterns in atypical parkinsonian syndromes, } \\
\text { for example midbrain predominant atrophy in PSP }\end{array}$ \\
\hline & CT brain & Calcium deposition in the basal ganglia (Fahr disease) \\
\hline \multirow{9}{*}{$\begin{array}{l}\text { Genetic } \\
\text { testing }\end{array}$} & DCTN1 & Perry syndrome \\
\hline & ATP13A2 & Kufor-Rakeb syndrome \\
\hline & FMR1 & Fragile X-associated tremor/ataxia syndrome \\
\hline & $\mathrm{HTT}$ & Huntington's disease with parkinsonism \\
\hline & CSF1R & $\begin{array}{l}\text { Adult-onset leukoencephalopathy with axonal spheroids and } \\
\text { pigmented glia }\end{array}$ \\
\hline & POLG & Mitochondrial disorder with parkinsonism \\
\hline & GBA & Gaucher's disease \\
\hline & NPC1 and NPC2 & Niemann-Pick C disease \\
\hline & CYP27A1 & Cerebrotendinous xanthomatosis \\
\hline
\end{tabular}

anti-Ri, anti-neuronal nuclear antibody type 2; ATP13A2, Gene, locus PARK9; CRMP5, collapsin response-mediating protein 5; CSF, cerebrospinal fluid; CSF1R, colony stimulating factor 1 receptor; CT, computed tomography; CYP27A1, cytochrome P450 family 27 subfamily A member 1; DCTN1, Gene encoding the dynactin subunit p150; DPPX, dipeptidyl-peptidase-like protein 6; FMR1, fragile $\mathrm{x}$ mental retardation 1; GBA, glucosidase beta acid; HIV, human immunodeficiency virus; HTT, Huntingtin gene; LGI1, leucine-rich glioma inactivated 1; Ma2, antiMa2; MRI, magnetic resonance imaging; NMDAR, N-methyl-D-aspartate receptor; NPC1, Niemann-Pick disease, type C1; NPC2, NiemannPick disease, type C2; PCR, polymerase chain reaction; POLG, DNA polymerase gamma gene; PSP, progressive supranuclear palsy.

is a potential cause of parkinsonism, withdrawing or changing potentially responsible medications is the first step. ${ }^{5}$ DIP usually resolves within months of stopping the offending drug but parkinsonism may persist or progress in $10 \%-50 \% .{ }^{5}$ While, unmasking of subclinical PD should be considered in this population, parkinsonism has been reported to persist for up to 18 months in some cases of DIP. $^{22}$

Despite medication withdrawal, patients can be significantly disabled by motor symptoms which may take time to resolve. There is also a risk of unmasking or exacerbating tardive dyskinesia with reduction or cessation of antipsychotics. ${ }^{17}$ Older and anecdotal evidence exists for the use of anticholinergic agents such as benztropine and levodopa in the symptomatic management of DIP, although it is not robust. ${ }^{25}$ Anticholinergic medications may also worsen concomitant tardive dyskinesia and amantadine has been proposed as a treatment option in this circumstance. ${ }^{17}$

Where antipsychotic medication is necessary, lowering the dose or switching to an antipsychotic with a lower risk of DIP such as quetiapine or clozapine is an alternative. ${ }^{5}$ Quetiapine is relatively safe in patients with parkinsonism and widely used for PD psychosis but has limited evidence for efficacy. ${ }^{34}$ Clozapine should be used judiciously given the potential for serious haematological and cardiac adverse reactions but, with adequate monitoring, is an effective treatment for psychosis ${ }^{35}$ with low risk for both parkinsonism and tardive dyskinesia. ${ }^{35} 36$ The increased mortality associated with antipsychotic use in older patients should also be carefully considered. ${ }^{37}$

Pimavanserin, a serotonin receptor inverse agonist shown to reduce psychotic symptoms in PD without worsening of motor symptoms, ${ }^{38}$ is not available for use in all countries.

If psychosis is secondary to a synucleinopathy such as PD or DLB, acetylcholinesterase inhibitors can be considered. Although not adequate for severe psychosis and with potentially limited approved indications, these agents can be antipsychotic sparing and have some evidence in PD psychosis. $^{11}$

In those with a primary psychiatric disorder, for whom electroconvulsive therapy can be prescribed, it has been shown to improve motor symptoms in both DIP and PD. ${ }^{39}$ 


\section{CONCLUSIONS}

In conclusion, the recognition, diagnosis and treatment of parkinsonism in patients with psychiatric conditions remains challenging. Research on clinical differentiation has aided identification but newer diagnostic investigations are not yet readily available. Underlying neurodegenerative pathology including PD pathology is more frequent in older patients who are therefore more susceptible in the psychiatric context to developing parkinsonism. The reported persistence of symptoms for up to 18 months in cases of DIP highlights the importance of making the diagnosis longitudinally. Cautious changes in medication regimens are required to treat parkinsonism where it arises in the psychiatric setting and new agents are not always necessary.

Acknowledgements We would like to thank Perminder Sachdev for his assistance in reviewing this manuscript.

Contributors AP was involved in the conception of the article, literature review and writing. LG was also involved in the conception and contributed to the literature review and writing. LK critically revised the draft manuscript. MWH was involved in the conception of the review and critically revised the draft manuscript.

Funding The authors have not declared a specific grant for this research from any funding agency in the public, commercial or not-for-profit sectors.

Competing interests None declared.

Patient consent for publication Not required.

Provenance and peer review Not commissioned; internally peer reviewed.

Open access This is an open access article distributed in accordance with the Creative Commons Attribution Non Commercial (CC BY-NC 4.0) license, which permits others to distribute, remix, adapt, build upon this work non-commercially, and license their derivative works on different terms, provided the original work is properly cited, appropriate credit is given, any changes made indicated, and the use is non-commercial. See: http://creativecommons.org/licenses/by-nc/4.0/.

ORCID iD

Alice Powell http://orcid.org/0000-0002-6146-420X

\section{REFERENCES}

1 Postuma RB, Berg D, Stern M, et al. MDS clinical diagnostic criteria for Parkinson's disease. Mov Disord 2015;30:1591-601.

2 Halliday J, Farrington S, Macdonald S, et al. Nithsdale schizophrenia surveys 23: movement disorders. 20-year review. Br J Psychiatry 2002;181:422-7.

3 Galvan A, Wichmann T. Pathophysiology of parkinsonism. Clin Neurophysiol 2008;119:1459-74.

4 Wichmann T. Changing views of the pathophysiology of Parkinsonism. Mov Disord 2019;34:1130-43.

5 Shin H-W, Chung SJ. Drug-induced Parkinsonism. J Clin Neurol 2012:8:15-21.

6 Schott JM. The neurology of ageing: what is normal? Pract Neurol 2017;17:172-82.

7 Estevez-Fraga C, Zeun P, López-Sendón Moreno JL. Current methods for the treatment and prevention of drug-induced parkinsonism and tardive dyskinesia in the elderly. Drugs Aging 2018;35:959-71.

8 Buchman AS, Yu L, Wilson RS, et al. Progressive parkinsonism in older adults is related to the burden of mixed brain pathologies. Neurology 2019;92:e1821-30.

9 Stahl SM. Stahl's essential psychopharmacology: neuroscientific basis and practical applications. New York: Cambridge University Press, 2011.

10 Ropper A, Samuels M, Klein J. Adams and victor's principles of neurology. 10th edn. USA: McGraw-Hill, 2014.

11 Ffytche DH, Creese B, Politis M, et al. The psychosis spectrum in Parkinson disease. Nat Rev Neurol 2017;13:81-95.

12 Stahl SM. Parkinson's disease psychosis as a serotonin-dopamine imbalance syndrome. CNS Spectr 2016;21:355-9.
13 Stahl SM. Mechanism of action of pimavanserin in Parkinson's disease psychosis: targeting serotonin $5 \mathrm{HT} 2 \mathrm{~A}$ and $5 \mathrm{HT} 2 \mathrm{C}$ receptors. CNS Spectr 2016;21:271-5.

14 Marston L, Nazareth I, Petersen I, et al. Prescribing of antipsychotics in UK primary care: a cohort study. BMJ Open 2014;4:e006135.

15 Dixit S, Khan SA, Azad S. A case of SSRI induced irreversible parkinsonism. J Clin Diagn Res 2015;9:VD01-2.

16 Hermida AP, Janjua AU, Glass OM, et al. A case of lithium-induced parkinsonism presenting with typical motor symptoms of Parkinson's disease in a bipolar patient. Int Psychogeriatr 2016;28:2101-4.

17 Ward KM, Citrome L. Antipsychotic-Related movement disorders: drug-induced parkinsonism vs. tardive Dyskinesia-Key differences in pathophysiology and clinical management. Neurol Ther 2018;7:233-48.

18 Martino D, Karnik V, Osland S, et al. Movement disorders associated with antipsychotic medication in people with schizophrenia: an overview of Cochrane reviews and meta-analysis. Can J Psychiatry 2018:730-9.

19 Shiroma PR, Geda YE, Mrazek DA. Pharmacogenomic implications of variants of monoaminergic-related genes in geriatric psychiatry. Pharmacogenomics 2010;11:1305-30.

20 Brigo F, Erro R, Marangi A, et al. Differentiating drug-induced parkinsonism from Parkinson's disease: an update on nonmotor symptoms and investigations. Parkinsonism Relat Disord 2014;20:808-14.

21 Hershey LA, Coleman-Jackson R. Pharmacological management of dementia with Lewy bodies. Drugs Aging 2019;36:309-19.

22 Gershanik OS. Drug-induced parkinsonism in the aged. recognition and prevention. Drugs Aging 1994;5:127-32.

23 Erro R, Bhatia KP, Tinazzi M. Parkinsonism following neuroleptic exposure: a double-hit hypothesis? Mov Disord 2015;30:780-5.

24 Tysnes O-B, Storstein A. Epidemiology of Parkinson's disease. J Neural Transm 2017;124:901-5.

25 López-Sendón JL, Mena MA, de Yébenes JG. Drug-induced parkinsonism in the elderly: incidence, management and prevention. Drugs Aging 2012;29:105-18.

26 Giladi N, Kao R, Fahn S. Freezing phenomenon in patients with parkinsonian syndromes. Mov Disord 1997;12:302-5.

27 Griffiths RI, Kotschet K, Arfon S, et al. Automated assessment of bradykinesia and dyskinesia in Parkinson's disease. J Parkinsons Dis 2012;2:47-55.

28 Deutschländer AB, Ross OA, Dickson DW, et al. Atypical parkinsonian syndromes: a general neurologist's perspective. Eur J Neurol 2018;25:41-58.

29 Bajaj N, Hauser RA, Grachev ID. Clinical utility of dopamine transporter single photon emission CT (DaT-SPECT) with (123l) ioflupane in diagnosis of parkinsonian syndromes. $J$ Neurol Neurosurg Psychiatry 2013;84:1288-95.

30 Goldstein DS. Dysautonomia in Parkinson's disease: neurocardiological abnormalities. Lancet Neurol 2003;2:669-76.

31 Bouwmans AEP, Vlaar AMM, Mess WH, et al. Specificity and sensitivity of transcranial sonography of the substantia nigra in the diagnosis of Parkinson's disease: prospective cohort study in 196 patients. BMJ Open 2013;3:e002613.

32 Schmidt MA, Engelhorn T, Marxreiter F, et al. Ultra high-field SWI of the substantia nigra at 7T: reliability and consistency of the swallowtail sign. BMC Neurol 2017;17:194.

33 Eratne D, Loi SM, Walia N, et al. A pilot study of the utility of cerebrospinal fluid neurofilament light chain in differentiating neurodegenerative from psychiatric disorders: $A$ ' $C$-reactive protein' for psychiatrists and neurologists? Aust N Z J Psychiatry 2020;54:57-67.

34 Rabey JM, Prokhorov T, Miniovitz A, et al. Effect of quetiapine in psychotic Parkinson's disease patients: a double-blind labeled study of 3 months' duration. Mov Disord 2007;22:313-8.

35 Parkinson Study Group. Low-dose clozapine for the treatment of drug-induced psychosis in Parkinson's disease. N Engl J Med 1999;340:757-63.

36 Sachdev PS. The current status of tardive dyskinesia. Aust N Z J Psychiatry 2000;34:355-69.

37 Weintraub D, Chiang C, Kim HM, et al. Association of antipsychotic use with mortality risk in patients with Parkinson disease. JAMA Neurol 2016;73:535-41.

38 Cummings J, Isaacson S, Mills R, et al. Pimavanserin for patients with Parkinson's disease psychosis: a randomised, placebocontrolled phase 3 trial. Lancet 2014;383:533-40.

39 Faber R, Trimble MR. Electroconvulsive therapy in Parkinson's disease and other movement disorders. Mov Disord 1991;6:293-303.

40 Balint B, Vincent A, Meinck H-M, et al. Movement disorders with neuronal antibodies: syndromic approach, genetic parallels and pathophysiology. Brain 2018;141:13-36. 